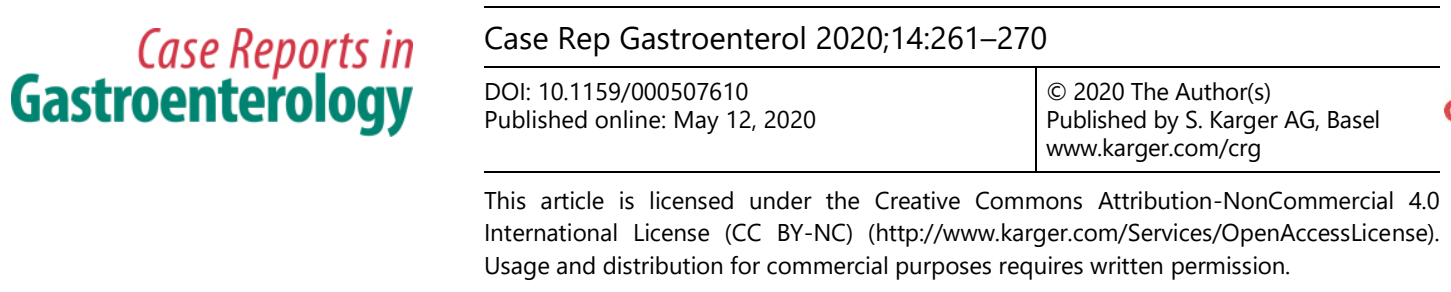

\title{
Over-the-Scope Clip to the Rescue! A Novel Tool for Refractory Acute Nonvariceal Upper Gastrointestinal Hemorrhage
}

\author{
Shivantha Amarnath $^{a} \quad$ Jobin Philipose $^{b}$ Jeffrey Abergel ${ }^{b} \quad$ Hafiz Khan $^{b}$ \\ aDepartment of Internal Medicine, Staten Island University Hospital, Northwell Health, \\ Staten Island, NY, USA; ${ }^{b}$ Department of Gastroenterology, Staten Island University \\ Hospital, Northwell Health, Staten Island, NY, USA
}

\section{Keywords}

Over-the-scope clip - Refractory gastrointestinal bleed - Upper gastrointestinal hemorrhage · Posterior duodenal wall ulcer · Nonvariceal bleed · Through-the-scope clip · Angiography

\begin{abstract}
Nonvariceal upper gastrointestinal hemorrhage (NVUGIH) is more prevalent than lower gastrointestinal hemorrhage and carries a high risk of mortality in the elderly, especially those with significant cardiovascular comorbidities. Traditional endoscopic methods, such as throughthe-scope clips, electrocautery, and epinephrine injection, are frequently used to control these bleeds; however, they carry a $10 \%$ risk of rebleeding, and this itself carries a mortality risk of $36 \%$. The larger over-the-scope clips (OTSC) that were initially used for the closure of fistulas and perforations are now gradually being implemented to manage NVUGIH. To our knowledge, we present the first cases to be reported in the literature where OTSC was successfully used as salvage therapy for refractory acute upper gastrointestinal bleeders who failed traditional endoscopic management and interventional radiology-guided embolization of the bleeding artery. We also provide an up-to-date literature review on the use of OTSC and its superiority to traditional endoscopic interventions in the management of complicated NVUGIH.




\section{Case Reports in Gastroenterology}

Case Rep Gastroenterol 2020;14:261-270

DOI: $10.1159 / 000507610$

(c) 2020 The www.karger.com/crg

Amarnath et al.: Over-the-Scope Clip to the Rescue! A Novel Tool for Refractory Acute Nonvariceal Upper Gastrointestinal Hemorrhage

\section{Introduction}

The annual incidence of gastrointestinal bleeding ranges from 20 to 170 cases per 100,000 per year. Upper gastrointestinal hemorrhage (UGIH) is 6 times more common than lower gastrointestinal hemorrhage. The former carries a high mortality risk, especially amongst the elderly population [1]. The annual incidence of hospitalization of UGIH in the USA is 400,000 per year [2]. Elderly patients $>70$ years of age with significant cardiovascular comorbidities are at a higher risk of nonvariceal UGIH (NVUGIH) compared to their healthier counterparts. For instance, the mortality rate of NVUGIH in those with cardiovascular disease (CVD) is $13.3 \%$ compared to $6.9 \%$ in those without CVD [3]. Control of bleeding in patients with NVUGIH is often achieved by conventional endoscopic methods, such as through-thescope clips (TTSC), electrocautery, and injection with epinephrine. However, these methods carry a $10 \%$ risk of recurrent bleeding, and this itself carries a mortality risk of $10-36 \%$. One of the critical risk factors for recurrent bleeding is the location of the ulcer, primarily the lesser curvature of the stomach and posterior duodenal abdominal wall.

Over-the-scope clip (OTSC), commonly referred to as the "bear claw," is a useful endoscopic clipping tool designed for tissue approximation. Despite its known efficacy in the closure of fistulas and perforations, it has now garnered popularity amongst endoscopists for its role in the management of NVUIGH. The barrel-shaped transparent cap provides adequate visualization of the lumen, maneuvering through challenging anatomic locations, and, ultimately, effective control of the bleeding. The device deploys a relatively large clip that compresses the vessels feeding a bleeding site. We posit that this modality can reduce the rate of rebleeding, especially bleeding from high-risk ulcers. Herein, we describe 2 high-risk elderly patients with American Society of Anesthesiology (ASA) physical status classification IV-V, who presented with NVUGIH due to a bleeding ulcer at the posterior duodenal bulb. These cases are unique in that they were deemed at high risk for any surgical intervention and had failed traditional endoscopic hemostasis modalities, as well as interventional radiology (IR)guided angiographic embolization of the gastroduodenal artery. To our knowledge, these are the first cases to be reported in the literature where OTSC was successfully used as salvage therapy for refractory gastrointestinal bleeders who failed IR-guided embolization. We also provide an up-to-date literature review on the successful management of OTSC and its superiority to traditional endoscopic interventions in the management of NVUGIH.

\section{Case Reports}

\section{Case 1}

An 85-year-old female with a history of atrial fibrillation (on Coumadin), pulmonary hypertension, and severe aortic stenosis presented with multiple episodes of hematemesis and melanic stools. Upon arrival, she was in hypovolemic shock with lactate of $5 \mathrm{mmol} / \mathrm{L}$. Her hemoglobin was $6.1 \mathrm{~g} / \mathrm{dL}$. She was resuscitated with blood products and intravenous fluids and was intubated and mechanically ventilated to protect her airways. Pantoprazole infusion was initiated, and Coumadin was held. The patient's Glasgow Blatchford Score (GBS) was 16, and pre-endoscopic Rockall score (RS) was 9. After volume resuscitation and stabilization, urgent upper endoscopy was performed. It revealed pooling of blood in the duodenum and multiple ulcers in the bulb (Fig. 1). Epinephrine was injected, and bipolar cautery was used to control the bleeding. The patient was returned to the intensive care unit for observation. 


\section{Case Reports in Gastroenterology}

Case Rep Gastroenterol 2020;14:261-270

DOI: $10.1159 / 000507610$

(c) 2020 The Author(s). Published by S. Karger AG, Basel www.karger.com/crg

Amarnath et al.: Over-the-Scope Clip to the Rescue! A Novel Tool for Refractory Acute Nonvariceal Upper Gastrointestinal Hemorrhage

Due to persistent melena, interventional radiologists performed angiography with embolization of the gastroduodenal artery (Fig. 2). Despite these efforts, the patient continued to bleed and was considered high risk for surgical intervention due to her pulmonary hypertension and valvular heart disease. Her hemoglobin dropped to $5 \mathrm{~g} / \mathrm{dL}$. A massive transfusion protocol was initiated. After a multidisciplinary discussion, a repeat endoscopy was performed with the utilization of the OTSC. During the procedure, a large blood clot was seen in the pylorus extending from an ulcer in the posterior aspect of the duodenal bulb. Epinephrine was injected at the crater of the ulcer and the clot was removed using a snare which exposed an actively bleeding vessel at the ulcer base (Fig. 3). An 11/6, T-type OTSC clip was deployed successfully, trapping the large visible vessel in the clip. The vessel was then further cauterized using a gold probe with successful hemostasis. No further bleeding was reported afterwards, and the patient remained stable. Four days after the second endoscopic intervention, the patient was discharged. The patient was followed up at 6 months, and she did not report any further episodes of bleeding.

Case 2

A 76-year-old bed-bound male with an extensive history of cardiac disease was brought to our hospital by emergency medical services after being rescued from a house fire. The patient had significant smoke inhalation injury and second-degree burns to his face, right arm, as well as his torso. He was intubated at the scene. He was admitted to the burn unit, underwent several surgeries for his burns, and eventually got tracheostomy placement due to respiratory failure. For nutritional support, the patient had an open gastrostomy tube placed by surgery. On day 6 of hospitalization, the patient developed melanic stools, and his hemoglobin dropped from a baseline of $10 \mathrm{~g} / \mathrm{dL}$ to $5.4 \mathrm{~g} / \mathrm{dL}$. He remained hemodynamically stable and was transfused appropriately with packed red blood cells. His GBS was 13, and pre-endoscopic RS was 7. He underwent endoscopy, which revealed that the PEG tube was in place without any surrounding injury to the stomach. There was also evidence of $3-\mathrm{cm}$ kissing ulcers in the duodenal bulb (Fig. 4). The posterior duodenal bulb ulcer had an actively bleeding visible vessel. Hemostasis was achieved with epinephrine injection, along with bipolar electrocautery, and the patient was monitored overnight in the intensive care unit.

Few hours after endoscopy, the patient developed melena with a drop in hemoglobin. He was resuscitated with fluids and massive blood transfusion. He then underwent urgent imageguided embolization of a suspected posterior gastroduodenal arterial bleed by IR (Fig. 5). Despite successful embolization of the bleeding vessel, the patient bled again. The patient was then taken for a second endoscopic intervention using OTSC. A large visible vessel was seen in the posterior wall of the duodenal bulb (Fig. 6). A 12/6, T-type OTSC clip was successfully deployed at the visible vessel in the posterior aspect of the duodenal bulb. The patient remained stable with a hemoglobin of $9 \mathrm{~g} / \mathrm{dL}$ for the remainder of the hospital course without any further evidence of bleeding. He was then discharged to a long-term facility. The patient was lost to follow-up.

\section{Discussion}

Traditional evidence-based endoscopic approaches used in the management of NVUGIH include TTSC, electrocautery, thermocautery, epinephrine injection, and use of hemostatic powders. However, these approaches are not without their limitations. The working channel of the traditional gastroscope is at the 7 o'clock position, so targeted therapy via the scope for 


\section{Case Reports in Gastroenterology}

Case Rep Gastroenterol 2020;14:261-270

DOI: 10.1159/000507610

(c) 2020 www.karger.com/crg

Amarnath et al.: Over-the-Scope Clip to the Rescue! A Novel Tool for Refractory Acute Nonvariceal Upper Gastrointestinal Hemorrhage

lesions located in the posterior duodenum or gastric cardia is challenging [4]. Application of TTSCs to large ulcers ( $>2 \mathrm{~cm}$ ), large visible vessels, or chronic ulcers with a fibrotic base can prove technically challenging. Additionally, multiple TTSCs are often required [1,4]. It is also particularly difficult in targeting bleeding sites in the posterior wall of the duodenum or lesser curvature of the stomach with a high failure rate [1]. Thermocoagulation can only effectively seal small bleeding arteries that are less than $2 \mathrm{~mm}$ [5]. Other limitations of the traditional approaches include, but are not limited to, dislodged stents and incomplete capture of the bleeding sites.

Despite traditional endoscopic management of NVUGIH, risk of rebleeding is $10-20 \%$, which carries a mortality risk of $10-36 \%[3,6]$. Major predictors of recurrent bleeding include advanced age, large ulcers $(>2 \mathrm{~cm}$ ), location of the ulcer (posterior duodenal wall or lesser curvature of the stomach), actively bleeding ulcer encountered during endoscopy, use of anticoagulants or antiplatelet agents, and comorbidities, such as end-stage renal disease [7]. Anatomic variation in blood supply to the duodenal bulb, either from branches of the celiac axis or superior mesenteric artery, may be the reason why our patients failed angiographic coil embolization of the gastroduodenal artery. The overall success of endoscopic retreatment to achieve hemostasis after an initial conventional endoscopic hemostatic intervention drops from 90 to $75 \%$ [6]. The OTSC is larger than standard hemostatic clips and, therefore, can encompass and approximate a large area of tissue or bleeding vessel with a high compression force of 8-9 Newtons. This reduces the need for the application of multiple clips and improves its initial success rate [3].

One of the initial prospective studies on the use of OTSC as first-line therapy for peptic ulcer bleeding came from Italy by Manno et al. [8]. They included 40 patients with a median age of 69 and with high-risk NVUIGH: 20\% gastric ulcers (Forrest IIa) and 18\% duodenal ulcers (Forrest Ib). Primary hemostasis was successfully achieved in all cases without any events of rebleeding or adverse outcome during the 30-day follow-up period.

A retrospective study from Singapore explored the effectiveness of OTSC in patients with high-risk adverse outcome (HR-AO) NVUGIH [9]. They defined HR-AO bleeding based on the following parameters: bleeding due to a large artery $>2 \mathrm{~mm}$; location within a major artery (left gastric and gastroduodenal artery); bleeding from a deeply penetrating, excavated or fibrotic ulcer; bleeding where management with endoscopic cautery incurred a high risk of perforation; location at pre-existing perforation sites, or when use of endoscopic mechanical therapy (TTSC) or radiological approach was unsuccessful. The standard of treatment of HR-AO bleeding includes use of cautery or hemostatic clips, but these have a failure rate of $20-40 \%$ [9]. The study included 18 patients with NVUGIH with a mean age of 68 years (50\% with ASA III), and $47 \%$ of these patients had bleeding duodenal ulcers. $53 \%$ of all bleeding patients had undergone OTSC as first-line management, and the remainder underwent OTSC as secondline treatment after an initial failed traditional endoscopic attempt. Interestingly, the rebleeding risk was reduced by 53\% $(p<0.01)$ in the OTSC group (high-risk pre-endoscopic Rockall group [RS >8]) as first-line therapy compared to those who underwent standard treatment. In the intermediate Rockall risk group (RS 4-7), OTSC reduced the risk by $24 \%(p<0.08)$. There was no difference in the risk of rebleeding in the low-risk Rockall group. There were at least 6 re-bleeders overall (33\% with duodenal ulcers), and complete hemostasis was achieved using OTSC as second-line therapy. The study established that OTSC can be safely used in patients with HR-AO lesions, even with a high-risk RS. It should be emphasized that the utilization of GBS or RS is only useful in risk stratifying patients for urgent endoscopic intervention and has not been validated for predicting clinical outcomes. 


\section{Case Reports in Gastroenterology}

Case Rep Gastroenterol 2020;14:261-270

DOI: 10.1159/000507610

2020 The www.karger.com/crg

Amarnath et al.: Over-the-Scope Clip to the Rescue! A Novel Tool for Refractory Acute Nonvariceal Upper Gastrointestinal Hemorrhage

To determine the effectiveness of OTSC in primary hemostasis of NVUGIH in elderly patients with severe CVDs and those on anticoagulation, a prospective study was conducted by Wedi et al. [3] in Germany. They included 100 patients with a median age of 72 years. 51\% of these patients had significant CVD, including congestive heart failure, valvular heart disease, peripheral arterial disease, and atrial fibrillation. 20\% had advanced kidney disease. $73 \%$ of these patients were on anticoagulation and/or antiplatelet therapy. The vast majority of these patients had an ASA classification of 3-5 (85\%). 79\% of these patients suffered from peptic ulcer bleeding, and of those, $54 \%$ were from the duodenum. The median size of the ulcer was $3 \mathrm{~cm}$, and $92 \%$ of these ulcers were high risk (Forrest Ia, Ib, and IIb). Using OTSC, primary hemostasis was achieved in $94 \%$ of NVUGI bleeders, and at 6 months follow-up, the incidence of rebleeding was $14 \%$. The failure rate was $<6 \%$. This was mostly due to difficulty visualizing the source of bleeding in 2 patients, and obstructing tumor in another. They also concluded that the NVUGIH mortality risk of $13.3 \%$ in elderly patients with CVD was reduced to $7.84 \%$ when using the OTSC approach.

The recent STING trial (a multi-center, randomized, unblinded, crossover study) by Schmidt et al. [6] was the first study to be conducted to directly compare OTSC with standard endoscopic therapy for recurrent bleeding from peptic ulcers. They incorporated 66 patients with recurrent NVUGIH after initial successful hemostasis via traditional endoscopy. Half of these patients underwent endoscopy directly using OTSC, and the remainder accepted the traditional approach, namely TTSC or cautery with injection with epinephrine. Patients who still had persistent bleeding despite standard therapy were allowed to cross over to the OTSC group. They found that OTSC achieved hemostasis without further bleeding in $84.9 \%$ of cases compared to $42.4 \%$ who underwent standard therapy $(p<0.001)$. There was also a relative risk reduction for further bleeding of $73.6 \%$. It should also be emphasized that $73 \%$ of the bleeding ulcers that were managed by OTSC were located in the posterior duodenal bulb, and hemostasis was achieved at a rate of $94 \%$ overall. The study had several limitations. The sample size was relatively small, and the study was open label. A vast majority of patients in the standard treatment arm primarily utilized TTSC and epinephrine rather than cautery. In terms of secondary endpoints, there was no significant difference between the OTSC arm and standard endoscopic therapy groups in terms of 30-day mortality or hospital stay.

Based on our literature review and clinical experience in the use of OTSC, we identified several shortcomings of the OTSC clip: deploying the device in the wrong location or shallow placement due to inadequate suction or premature clip deployment; difficulty in introducing the probe in intubated patients or in those with stenosis along the alimentary tract; mucosal injury during insertion; and the need for withdrawal and reintroduction of the scope to deploy the clip after identifying the target site which may be actively bleeding $[9,10]$. The risk of deployment failure can be reduced by ensuring adequate suction of sufficient tissue at the bleeding site into the cap and maintaining a clear view of the lumen when possible. Utilization of the OTSC at the site of the posterior duodenal bulb is not without its challenges; the relatively narrow lumen of the duodenum with thin walls makes it difficult to access and maintain proper position. There is also a higher risk of perforation in the duodenum as compared to other parts of the upper gastrointestinal tract. Certain scenarios can prove even more technically challenging: the retroflexed position, angulated parts of the intestinal tract, large fibrotic ulcers, and complex postsurgical anatomy. Therefore, it is imperative that any gastroenterologist and fellows who plan on using OTSC ensure that they have achieved adequate training.

These 2 high-risk cases demonstrate the effectiveness of the OTSC to achieve hemostasis in anatomically challenging ulcers, especially those located in the posterior duodenal wall. It must also be emphasized that the OTSC was successful as salvage therapy when conventional 
endoscopic management using TTSC and IR embolization of the bleeding vessel had failed. One could extrapolate that the utilization of OTSC may reduce the length of hospital stay and the need for recurrent blood transfusion or surgery. However, we did not come across any literature documenting this. No trials have been conducted thus far comparing OTSC with surgical or radiological intervention in the control of NVUGIH after a failed conventional endoscopic intervention. Further trials would need to be performed to demonstrate the level of safety and effectiveness of OTSC in bleeding patients on anticoagulant therapy. More studies need to be carried out with emphasis on these outcomes with a direct head-to-head comparison between OTSC and a traditional endoscopic approach to hemostasis. Currently, a randomized controlled trial referred to as the CURE trial is underway to compare OTSC with standard endoscopic hemostasis using epinephrine injection, multipolar coagulation, and TTSC, thereby looking at the rebleeding rate of NVUGIH at 30 days [11].

\section{Acknowledgement} history.

We would like to thank the patients for allowing us to share the details of their clinical

\section{Statement of Ethics}

We hereby confirm that informed consent was obtained from the patients for publication of the case details.

\section{Disclosure Statement}

The authors have no conflicts of interest to declare.

\section{Funding Sources}

There are no financial disclosures or funding sources.

\section{Author Contributions}

Shivantha Amarnath, MD: literature research and writing the manuscript. Jobin Philipose, MD: editing and reviewing the manuscript. Jeffrey Abergel, MD, FACG: study mentor and critical revision of the manuscript. Hafiz Khan, MD: study mentor and critical revision of the manuscript.

\section{References}

1 Manta R, Mangiafico S, Zullo A, Bertani H, Caruso A, Grande G, et al. First-line endoscopic treatment with over-the-scope clips in patients with either upper or lower gastrointestinal bleeding: a multicenter study. Endosc Int Open. 2018 Nov;6(11):E1317-21. 


\section{Case Reports in Gastroenterology}

Case Rep Gastroenterol 2020;14:261-270

DOI: $10.1159 / 000507610$

(c) 2020 The Author(s). Published by S. Karger AG, Basel www.karger.com/crg

Amarnath et al.: Over-the-Scope Clip to the Rescue! A Novel Tool for Refractory Acute Nonvariceal Upper Gastrointestinal Hemorrhage

2 Park T, Wassef W. Nonvariceal upper gastrointestinal bleeding. Curr Opin Gastroenterol. 2014 Nov;30(6):603-8.

3 Wedi E, von Renteln D, Gonzalez S, Tkachenko O, Jung C, Orkut S, et al. Use of the over-the-scope-clip (OTSC) in non-variceal upper gastrointestinal bleeding in patients with severe cardiovascular comorbidities: a retrospective study. Endosc Int Open. 2017 Sep;5(9):E875-82.

4 Martínez-Alcalá A, Mönkemüller K. Emerging Endoscopic Treatments for Nonvariceal Upper Gastrointestinal Hemorrhage. Gastrointest Endosc Clin N Am. 2018 Jul;28(3):307-20.

5 Agarwal AK, Miglani S, Singla S, Garg U, Dudeja RK, Goel A. Ultrasonographic evaluation of gallbladder volume in diabetics. J Assoc Physicians India. 2004 Dec;52:962-5.

6 Schmidt A, Gölder S, Goetz M, Meining A, Lau J, von Delius S, et al. Over-the-Scope Clips Are More Effective Than Standard Endoscopic Therapy for Patients With Recurrent Bleeding of Peptic Ulcers. Gastroenterology. 2018 Sep;155(3):674-686.e6.

7 Elmunzer BJ, Young SD, Inadomi JM, Schoenfeld P, Laine L. Systematic review of the predictors of recurrent hemorrhage after endoscopic hemostatic therapy for bleeding peptic ulcers. Am J Gastroenterol. 2008 Oct;103(10):2625-32; quiz 2633.

8 Manno M, Mangiafico S, Caruso A, Barbera C, Bertani H, Mirante VG, et al. First-line endoscopic treatment with OTSC in patients with high-risk non-variceal upper gastrointestinal bleeding: preliminary experience in 40 cases. Surg Endosc. 2016 May;30(5):2026-9.

9 Asokkumar R, Soetikno R, Sanchez-Yague A, Kim Wei L, Salazar E, Ngu JH. Use of over-the-scope-clip (OTSC) improves outcomes of high-risk adverse outcome (HR-AO) non-variceal upper gastrointestinal bleeding (NVUGIB). Endosc Int Open. 2018 Jul;6(7):E789-96.

10 Goenka MK, Rai VK, Goenka U, Tiwary IK. Endoscopic Management of Gastrointestinal Leaks and Bleeding with the Over-the-Scope Clip: A Prospective Study. Clin Endosc. 2017 Jan;50(1):58-63.

11 ClinicalTrials.gov [Internet]. Bethesda, MD: National Library of Medicine (US); 2000 Feb 29. Identifier NCT03216395. Over-the-scope Clips and Standard Treatments in Endoscopic Control of Acute Bleeding from Non-variceal Upper GI Causes; 2019 June 10 [cited 2019 Sep 1]. Available from: https://clinicaltrials.gov/ct2/show/record/NCT03216395

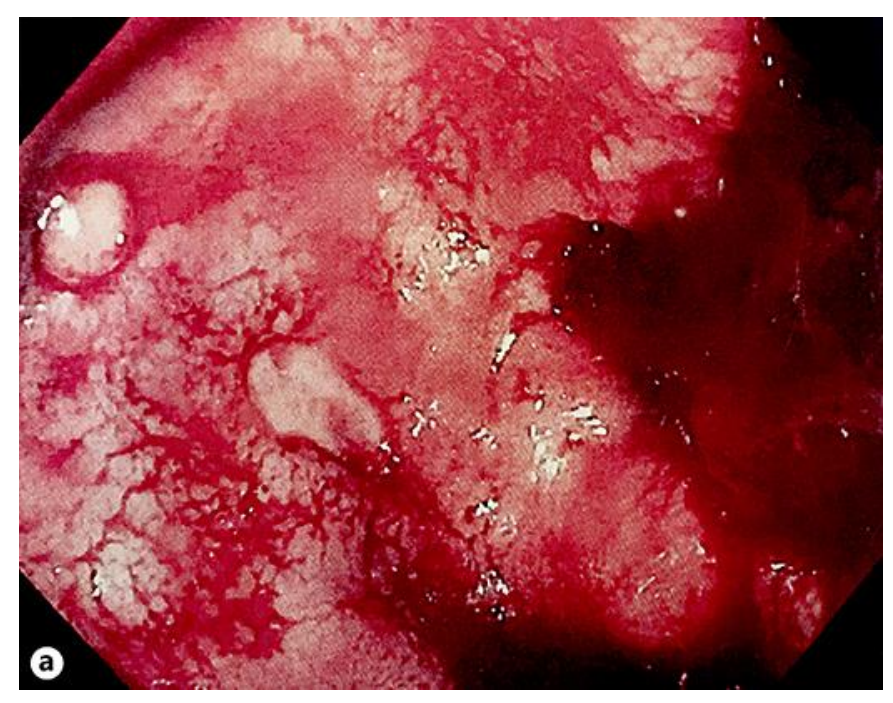

Fig. 1. Duodenal bulb ulcer with clot (Forrest IIb). 

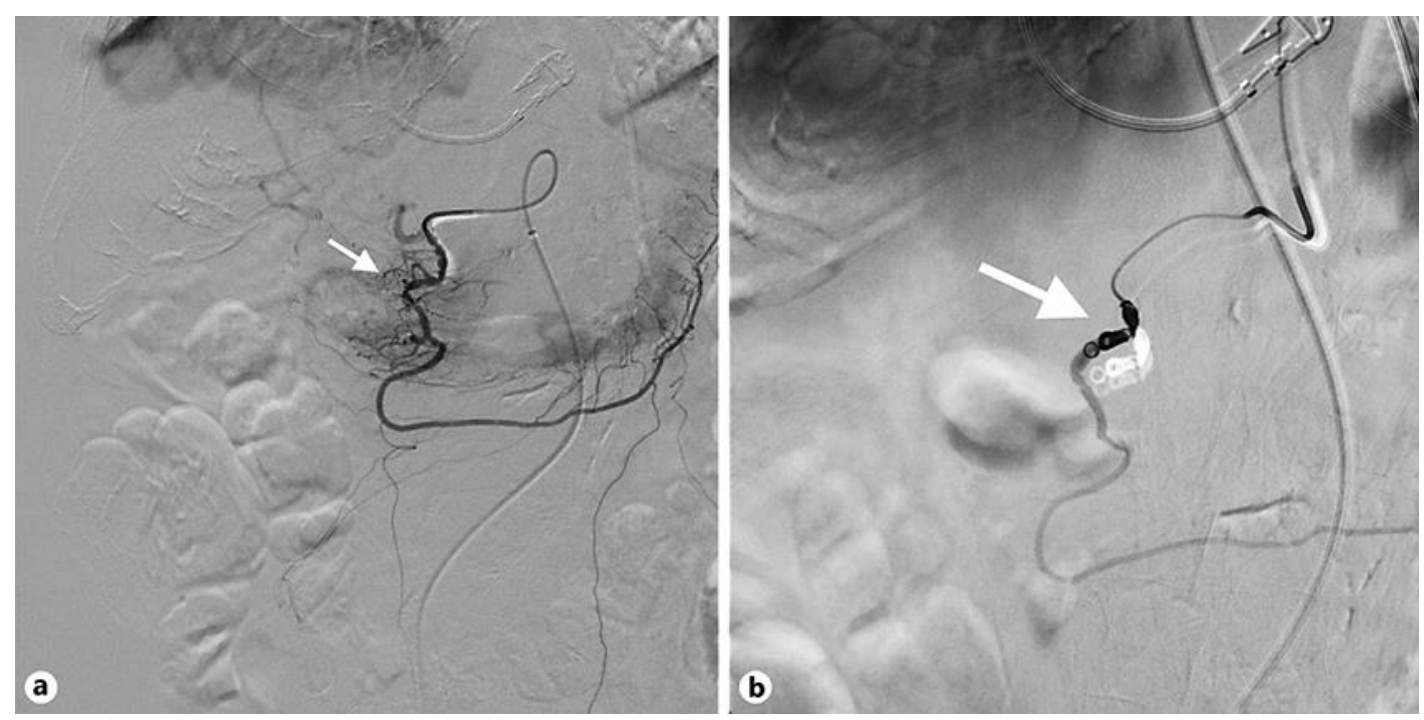

Fig. 2. Transcatheter angiography of the celiac artery with active contrast extravasation in multiple small branches of the proximal posterior gastroduodenal artery (a). Coil embolization of the proximal gastroduodenal artery with 2 micro-coils (b). 


\section{Case Reports in Gastroenterology}

\begin{tabular}{l|l}
\hline Case Rep Gastroenterol 2020;14:261-270 \\
\hline DOI: 10.1159/000507610 & $\begin{array}{l}\text { @ 2020 The Author(s). Published by S. Karger AG, Basel } \\
\text { www.karger.com/crg }\end{array}$ \\
\hline
\end{tabular}

Amarnath et al.: Over-the-Scope Clip to the Rescue! A Novel Tool for Refractory Acute Nonvariceal Upper Gastrointestinal Hemorrhage
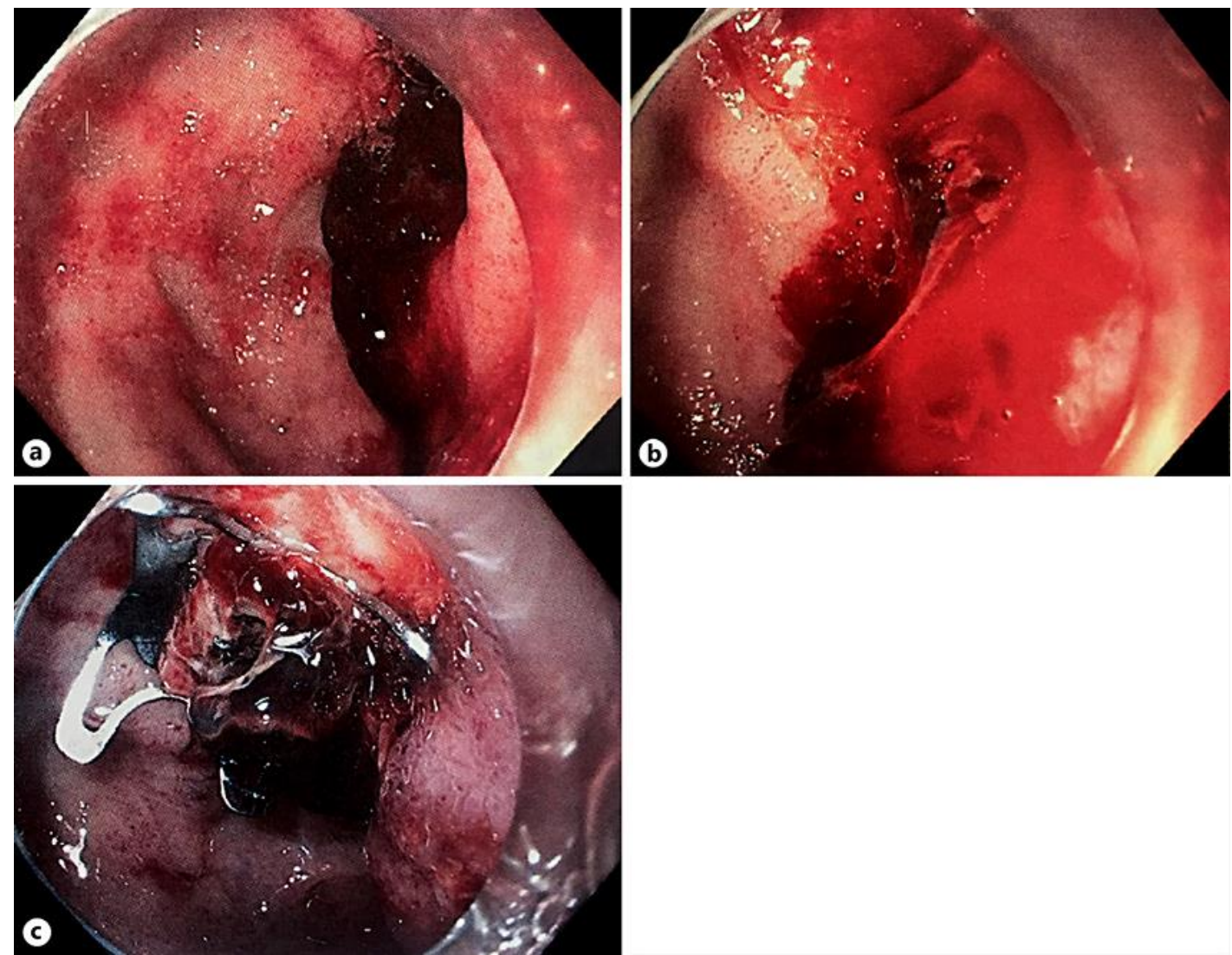

Fig. 3. Posterior duodenal bulb ulcer with adherent clot (a). Actively bleeding vessel (Forrest Ia) in the posterior duodenal ulcer (b). OTSC clip application on the actively bleeding vessel with successful hemostasis (c).
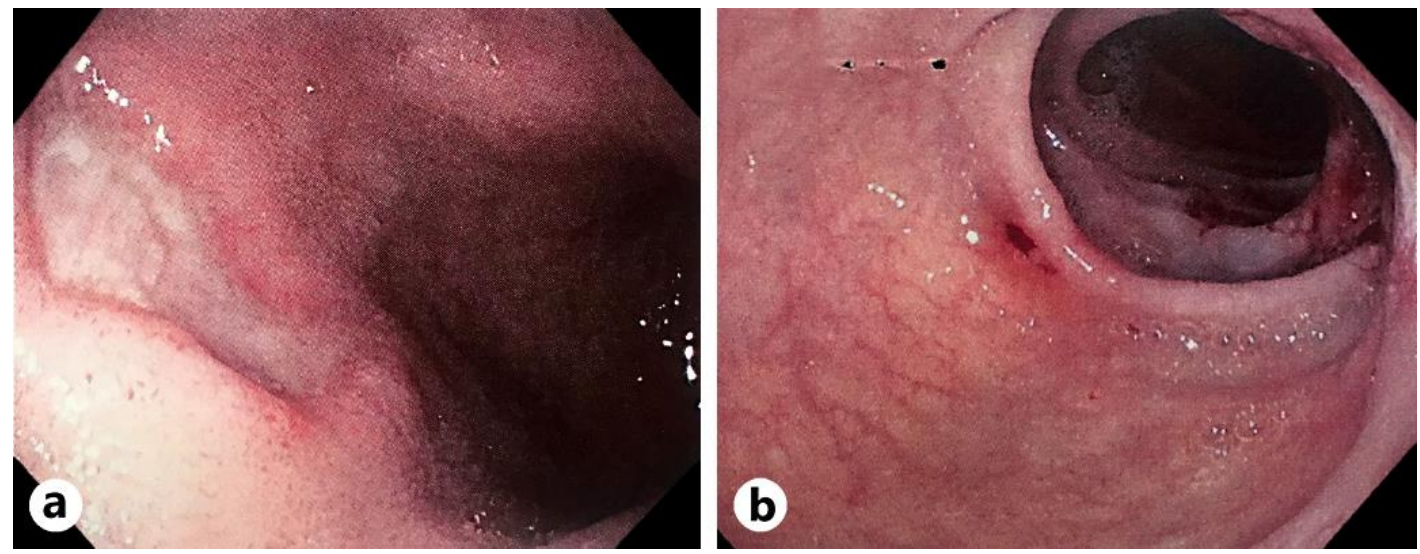

Fig. 4. Large 3-cm clean based ulcer in the anterior duodenal bulb (Forrest III) (a). 2-cm ulcer in the posterior duodenal bulb with a visible vessel (Forrest Ib) (b). 


\section{Case Reports in Gastroenterology}
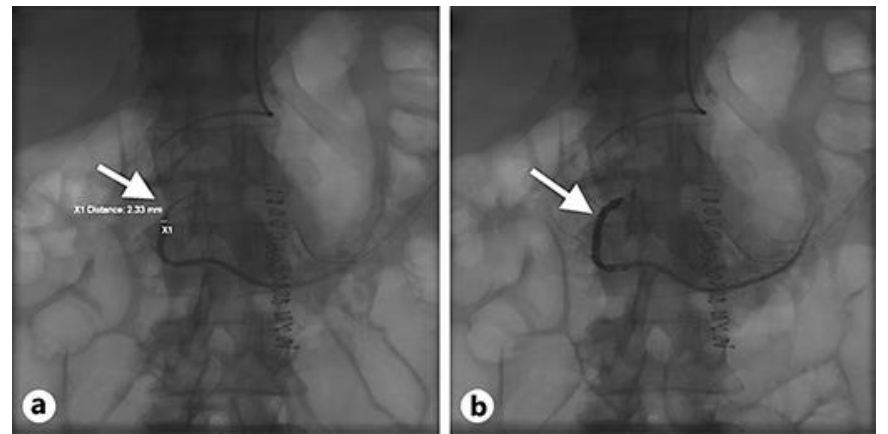

Fig. 5. Transcatheter angiography of the gastroduodenal artery demonstrating vasospasm without evidence of active contrast extravasation or pseudoaneurysm (a). Prophylactic coil embolization of the gastroduodenal artery (b).

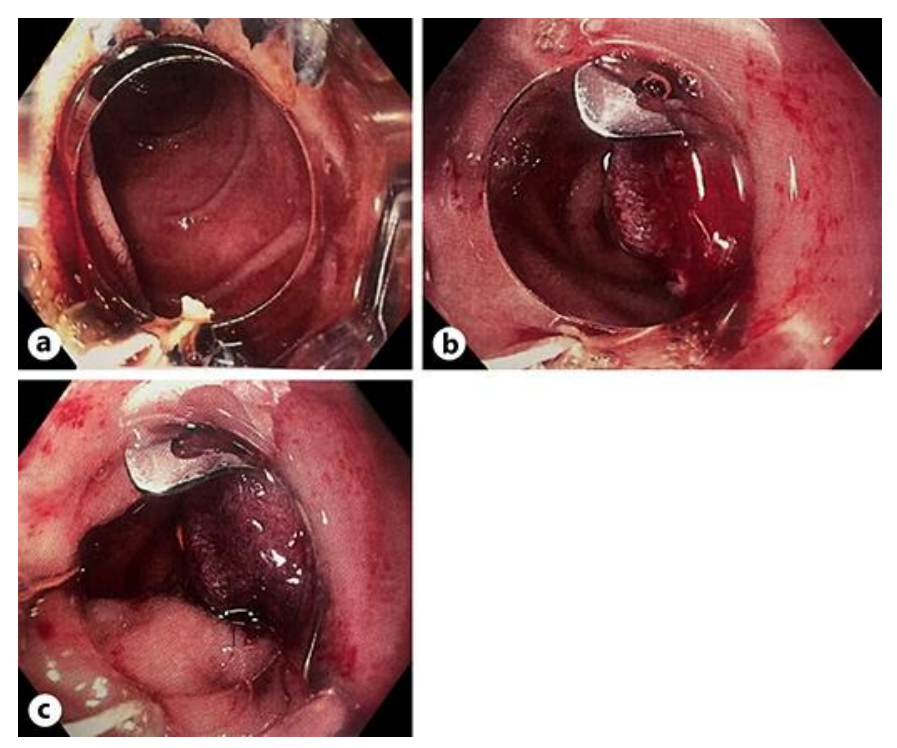

Fig. 6. OTSC mounted over the scope for deployment (a). Successful deployment of a 12/6 OTSC on the visible vessel in the posterior duodenal bulb (b, c). 